\title{
Reservation Enhanced Autonomous Valet Parking Concerning Practicality Issues
}

\author{
Xu Zhang, Fang Yuan, Yue Cao and Shuohan Liu
}

\begin{abstract}
Advances in automotive industry as well as computing technology are making autonomous vehicle (AV) an appealing means of transportation. Vehicles are beyond the traditional source of commute, and leveled up to smart devices with computing capability. As one of the compelling feature of AVs, the autonomous valet parking (AVP) allows for navigating and parking the car automatically without human interventions. Within this realm, Long-range AVP (LAVP) extends auto-parking to a much larger scale compared to its short-range counterparts. It is worth noting that $\mathrm{AV}$ mobility is a pivotal concern with LAVP, involving dynamic patterns related to spatial-temporal features, such as varied parking and drop-off (or pick-up) demands with diverse customer journey planning. We herein target such critical decision-makings on where to park and where to drop/pick-up upon customer requirements during their journeys. Concerning in practice that car parks are equipped with limited parking space, we thus introduce parking reservations and enable accurate estimations on future parking states. An efficient LAVP service framework enhanced with parking reservations is then proposed. Benefited from the intelligent predictions, parking load can be accurately predicted and greatly alleviated at individual car parks, thereby avoiding overcrowding effectively. Results show that significant performance gains can be achieved under the proposed scheme by comparing to other benchmarks, with respect to greatly reduced waiting duration for available parking space, as well as enhanced customer experiences in terms of reduced travelling period, etc. In particular, the number of parked vehicles across the network can be effectively balanced.
\end{abstract}

Index Terms-Autonomous vehicle, Autonomous valet parking, Transportation planning.

\section{INTRODUCTION}

O VER the last few decades, vehicle ownership rates have grown exponentially as their costs decrease. Meanwhile, the rapid rise in automobiles has imposed a range of challenges, from traffic congestion to environmental pollution [1]. This creates an urgent need for more efficient resources and infrastructure support, so as to accommodate such enormous increase in the rate of adoption of autos [2]. Therefore, the automotive industry has been striving to achieve these expectations by seeking for technologically advanced, fully automated

$\mathrm{Xu}$ Zhang is with the Department of Computer Science and Engineering, Xi'an University of Technology, Xi'an, 710048, China, e-mail: zhangxu@xaut.edu.cn

Fang Yuan is with College of Automotive Engineering, Dalian University of Technology, 116024, China, e-mail: yuanfang_sophie@163.com

Y.Cao (corresponding author) is with the School of Cyber Science and Engineering, Wuhan University; Shenzhen Key Lab of Data Visualization (Smart City), Research Institute of Beihang University in Shenzhen, China. Email: 871441562@qq.com

S.Liu is with School of Computing and Communications, Lancaster University, LA1 4YW, UK, e-mail: liu37@lancaster.ac.uk

The research is supported in part by the Joint Fund of Guangdong Province Foundation and Applied Science (2019A1515110238). and reliable means of transportation, and the autonomous vehicle (AV) [3] starts to thrive.

Because of unprecedented development in motor manufacturing and computing technology, the purely mechanical source of commute has begun to shift into a smart and infotainment-rich computing and transportation device on-themove. With computer-aided control, an autonomous car can fully operate without any human interaction. Traffic collisions caused by human errors can therefore be substantially reduced when automated vehicles are maturely developed. One immediate advantage of automated cars is to replace behind-thewheel commuting hours with more time for leisure or work. As a key capability of AVs, the autonomous valet parking (AVP) [4] would provide another compelling feature: the ability to locate and park the car without human interactions. Further, AVP would allow for passengers drop-off and pick-up in places where longtime parking is prohibited.

As reported, car parking is a major issue in urban areas, and vehicles are being parked and unused for most of the time [5]. Within this realm, AVP relieves travelers from navigation and maneuvering chores, and could dramatically reduce the need for parking space. For example, parking is completely automatic and problem-free under AVP even if space is tight, which could benefit park and ride facilities.

There have been a few work and research put into this concept of AVP over the years, most of which focus on Short-range AVP (SAVP) [6][7][8]. By leveraging vision and sensor system along with advanced control techniques, the SAVP system is able to locate a free space in the parking garage and parks the car autonomously by means of connected technology [9]. In this situation, the vehicle performs the parking maneuvers without human intervention, usually after a learning process guided by the driver about the environment and trajectories. Due to limitations on the short-range effectiveness, the SAVP starts to work from at the entrance of a park.

As of yet, much less attention has been given to the Longrange AVP (LAVP), which extends the auto-parking to a much larger scale. Within this concept, the vehicle is able to drop-off/pick-up drivers close to their final destinations (e.g., work places) rather than at the parking lot entrance. The AV would then familiarize itself with surroundings [10] and continually drive and park without any human interaction. It is worth noting that $A V$ mobility is a pivotal concern with LAVP, involving dynamic patterns related to spatial-temporal features, such as fuel consumption and several key decisionmakings (e.g., where to park and where to drop/pick-up) with varied customer demands. 
With LAVP, procedures related to selection of drop-off/pickup points as well as choice on car parks are among the major concerns. Even though, limited research efforts have been put forth into this realm [11][12]. Particularly, considering limited parking space in practice, a parking lot can quickly become overcrowding due to unexpected huge arrivals of AVs in a short time. Long queues could otherwise cause congestions where autos can be left stranded. Such concerns have not yet been well addressed in literatures within LAVP. With this insight, our technical contributions are thus as the following:

1) Enabling LAVP with accurate parking predictions: Considering the practical concern on the inadequate parking space, it requires to select car parks more intelligently. By introducing parking reservation service ${ }^{1}$ prior to car parking while AV on the go, parking states in a future moment across all car parks can be accurately estimated, thereby alleviating parking hotspots effectively.

2) An efficient design of LAVP service framework concerning parking capacity: In order to provide the optimal car park for the vehicle, as well as the most appropriate drop-off/pickup point for customers, a range of critical context needs to be considered. They include travel distance, the potential overcrowding at car parks and the customer experiences, etc. These factors all play a pivotal role in the design of an efficient LAVP service. By accounting for a joint concern on these critical context information, an efficient LAVP service framework is developed to achieve such aim, in terms of minimized vehicle trip duration and car park waiting time, as well as enhanced customer experiences (in terms of customer travel period).

\section{BACKGROUND}

The autonomous car has received a lot of attention during the past decade and prototype versions have been developed by different vendors. Recent developments in vehicular adhoc network (VANET) and connected car technologies have attracted the attention of companies such as Google in the development of driverless cars [13][14][16]. In addition to Google, car manufacturers such as Tesla and Audi are two leading stakeholders in driverless car technology [17]. In the Asian market, TATA, Yutong, KIA, and Hyundai are major companies investing in the autonomous cars design, development, and research [18].

\section{A. AVP Service}

As one appealing feature, AVP is an important milestone paving the way towards vehicle automation, which has drawn wide attention from industries and research communities. Leading technical companies and car manufacturers are actively engaged into this realm. Specifically, a joint pilot project of Mercedes-Benz and Bosch are in close cooperation to develop such innovative parking service [19]. The system is able to locate a free space at a parking garage and parks the vehicle autonomously through connected technology. An

\footnotetext{
${ }^{1}$ A parking reservation includes the information such as the expected arrival time of the vehicle, as well as for how long the car will be parked.
}

experimental car equipped with AVP functionality has also been tested in [4]. During their trials, there is a learning process for the vehicle before it is able to park itself. Namely, the vehicle learns the surroundings and trajectories while the driver performs the usually driving process manually (after entering the garage). Only after the learning process, the vehicle can finish parking autonomously.

Within the area of AVP, it is worth noting that most studies focus on managing parking within or in proximity to a car park [6][7][8], usually known as the SAVP. The technology allows drivers to be dropped off at/near the entrance of a parking facility, and then the vehicle navigates and parks itself with the aid of indoor parking maps. To get back to the vehicle, the user would summon it via a smartphone app. To enable such automation executions, it requires the car to be capable of gaining perception of the environment in terms of real-time mapping acquisition, and the ability to generate a trajectory to adapt to in real-time, etc. By the means of connected and sensing technology, e.g., vehicle to infrastructure (V2I) communication techniques, a cooperative vehicle control algorithm can be applied in AVP as proposed in [15], to control the vehicle forward and backward driving in a parking lot.

\section{B. LAVP Service}

In urban areas, car parks are usually located outside of city centers to avoid traffic congestions and high-cost land use. From the perspective of AV drivers, drop-off/pick-ups are preferred to happen at places close to their destinations (e.g., work places), which are usually located close to city centers. Therefore, recent research efforts are staring to extend the scope of AVP to a much larger scale, namely the LAVP [11][12]. In this case, a drop-off spot might be distant away from the car park. The AVP begins from the drop-off point, and the vehicle autonomously finds and navigates to parking. Meanwhile, the user would walk (or by other means of transportation) towards his/her destination from that spot.

However, because of the mobility nature of vehicles, the design of LAVP concerning the overall journey experience remains a significant challenge [8]. As previously noted, several important issues remain, such as travel cost and route selection (concerning drop/pickup point-selection and car parkselection), which requires efficient solutions for LAVP.

\section{Our Motivation}

Driven by rising interests in LAVP, we herein focus on the design of an efficient LAVP framework in this paper. Particularly, concerning the limited parking capacity in practice, we introduce parking-reservation into LAVP to avoid parking congestions. While most existing research efforts are given to SAVP, attention on LAVP is lacking. We hope the design of this work will help utilities in the realm of AVP where there would be potential reduced need for road space due to improved traffic flow, thereby freeing up tremendous amounts of land in urban areas for other usage. 


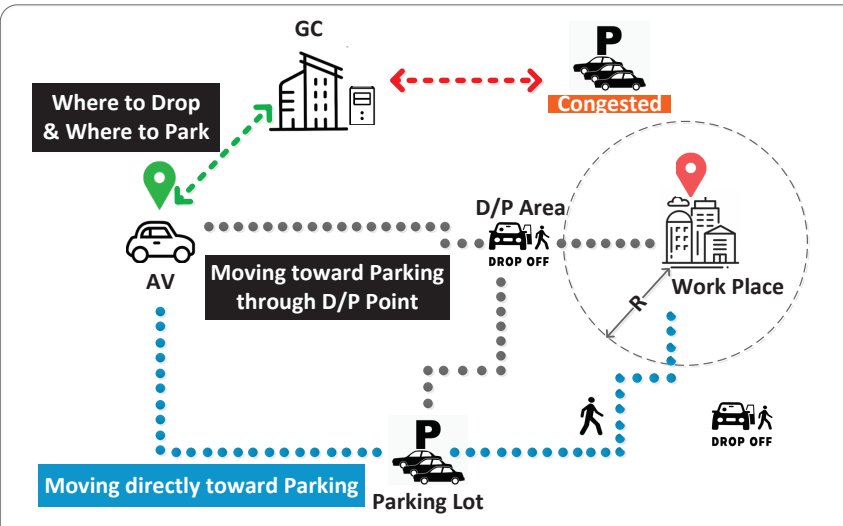

$\ldots \ldots . . . . \Rightarrow$ Communication between GC and AV $\quad \ldots \ldots$ LAVP Mode $\ldots . . . . \rightarrow$ Communication between GC and CP .... Traditional AVP Mode

Fig. 1. Big picture of LAVP where an AV selects optimal D/P point and parking lot with the aid of a cloud server GC

\section{RESERVATION ENHANCED LAVP SERVICE}

\section{A. Overview}

LAVP provides the capability for automobiles to drive and park independently when parking is needed. As depicted in Fig. 1, An AV (i.e., $a v_{r}$ ) is traveling towards the customers destination, e.g., to his/her work place (WP). While on the move, the vehicle $\left(a v_{r}\right)$ may send a parking request to a cloudbased global controller (GC) for best parking lot suggestion. The GC processes parking requests from $\mathrm{AVs}$ and would reply a best-choice of drop-off/pick-up (D/P) spot close to the customers final destination. Once the D/P-selected is confirmed by the $a v_{r}$, the GC would then recommend an optimal car park (CP) for the $\mathrm{AV}$, which should not be too far from the $\mathrm{D} / \mathrm{P}$ point. A parking reservation is then reported if the CP suggestion is accepted. Note that the D/P-selected would be the place where customer is dropped/picked-up ${ }^{2}$. From that area, the customer will walk (or take other forms of transportation) toward the destination (i.e., WP), and ask for a pick-up later on. Meanwhile, the AV automatically navigates itself toward the reserved $\mathrm{CP}$ and parks itself. In a conventional AVP case, the driver needs to come along with AV towards CP, before walking all the way to the final destination (i.e., WP), where none D/P points involved. Specifically, the following network entities are involved in a LAVP service:

- Autonomous Vehicle (AV): While traveling, an AV may need to park anytime during the journey, due to varied customer demands. Considering the final destination of the customer, the most appropriate $\mathrm{D} / \mathrm{P}$ spot needs to be selected concerning the walk distance for the customer. Given the distribution of D/P spots that could be dispersed around that area (i.e., the customers final destination), energy consumption of the vehicle also plays a key role

\footnotetext{
${ }^{2}$ In practice, the drop off area may differ from the pick-up point owing to various demands from customers. In this work, we assume D/P points are at the same location to simplify the analysis. However, our policy proposed in this paper can apply well in scenarios where D/P spots locates differently.
}

in selecting appropriate $\mathrm{D} / \mathrm{P}$ point. Once the $\mathrm{D} / \mathrm{P}$ spot is confirmed, the customer will be dropped off there, and the AV would then find somewhere proper to park while the customer is staying at the destination. Once needed, the AV would travel back to the $\mathrm{D} / \mathrm{P}$ point to pick up the customer. Note that the LAVP starts to operate from D/P spot all the way towards the CP.

- Car Park (CP): Each CP holds a number of parking spots, and the amount (defined as $C_{P}$ ) is finite due to limitations on space. The status regarding parking availability is monitored in real-time by the GC. In mega cities, CPs usually locate in suburbs of a city for the purpose of large parking capacity. Although a CP can be large enough for hundreds of vehicles, the $\mathrm{CP}$ can become saturated quickly with constant AV arrivals, however. Therefore, a parking reservation is suggested prior to the arrival of an AV in the system.

- Global Controller (GC): The GC monitors CP parking conditions through cellular networks, and processes parking requests from $\mathrm{AVs}$. As previously discussed, the GC determines the best D/P point as well as the optimal $\mathrm{CP}$, through intelligent selection decision-makings, which will be elaborated in Sec. IV. It is worth noting that the selection on $\mathrm{D} / \mathrm{P}$ spot can be bounded within a certain range, e.g., within a radius of $R$ of the final destination (as shown in Fig. 1). With respect to $\mathrm{CP}$-selection, the travel distance (from D/P point to $\mathrm{CP}$ ) as well as the parking status (i.e., parking availability) is among the key concerns.

As for $\mathrm{D} / \mathrm{P}$ points, they are locations where temporary parking is allowed, e.g., for minutes only. As such, the flow of traffic tend to move smoothly and hence, D/P zones are normally treated as non-congestion areas. Therefore, D/P locations and their distances towards customers destinations are among major concerns for D/P-selection decision-making, except for traffic conditions.

\section{B. Protocol for Reservation Enhanced LAVP Service Process}

The communication in Intelligent Transportation System$\mathrm{s}$ (ITS) can be leveraged by AVs to enable information broadcasting between involved entities, through the ubiquitous cellular networks. AVs can also be built with GPS sensing knowledge to help with navigation. We herein discuss the communication signaling among main network entities, and Fig. 2 describes the time sequences.

Step 1 Real-time monitoring: The GC keeps track on all CPs of their parking status, including the number of parked vehicles, their expected parking period and parking reservations. Meanwhile, traffic information around the $\mathrm{D} / \mathrm{P}$ area will be updated to GC in real-time (typically periodically), so as to inform the AV to try to avoid congested street areas.

Step 2 Reporting parking request: While on the move, $a v_{r}$ may send a parking request to $\mathrm{GC}$ for optimal selections on $\mathrm{D} / \mathrm{P}$ spots and CPs.

Step 3 Best D/P point-selection: Upon receiving the parking enquiry from a vehicle, GC executes the $\mathrm{D} / \mathrm{P}$ point-selection process (according to Alg. 1 as will be stated in Sec. IV), and 


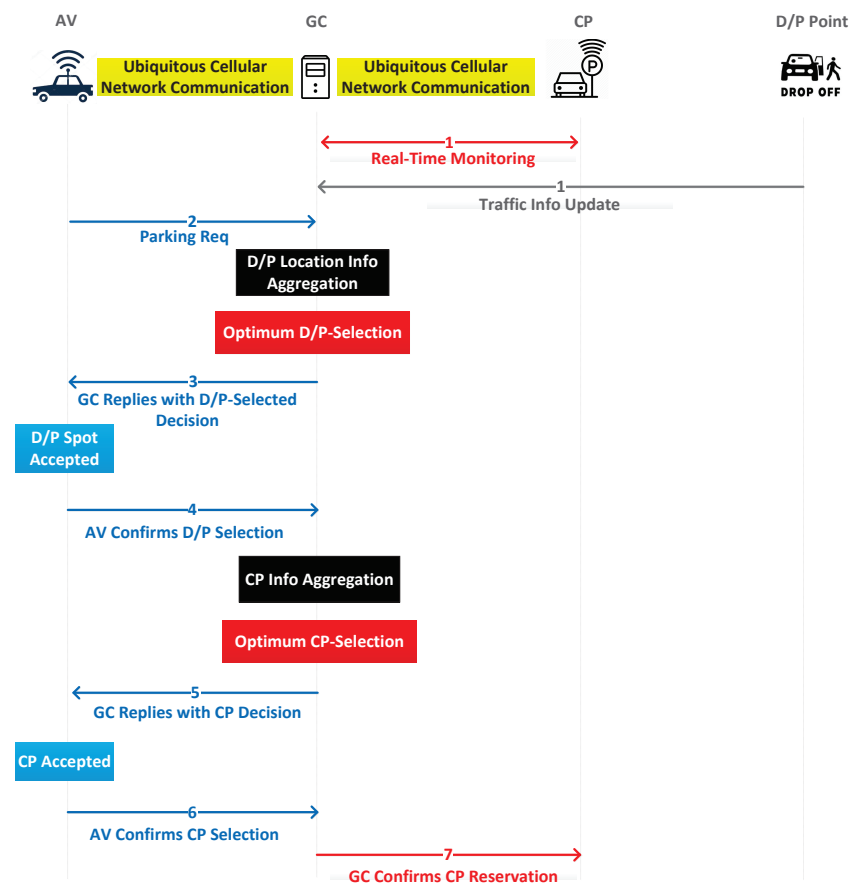

Fig. 2. Time sequences of Reservation Enhanced LAVP

selects the most appropriate one. The D/P-selection decision would then be replied to the requestor vehicle $a v_{r}$.

The decision-making on D/P-selection targets minimizing the outbound (and inbound) trip duration for the customer, including a joint concern on the travel time of $a v_{r}$ (from its current location to the $\mathrm{D} / \mathrm{P}$ point), as well as the walk time for the customer (From D/P point to the destination).

Step 4 Reporting confirmation on D/P-selected: Notified by the GC, $a v_{r}$ confirms the D/P-selection decision by sending back its reply. In case that $a v_{r}$ denies the suggestion, GC keeps searching for a new $\mathrm{D} / \mathrm{P}$ point until the arrangement is accepted.

Step 5 Optimal CP-selection: Once GC receives the D/P confirmation from $a v_{r}$, it starts to compile a list of CPs by accounting for the vehicles travel distance to a $\mathrm{CP}$, along with the parking availabilities at individual CPs (such decisionmaking process is based on Alg. 4 in Sec. IV). An optimal $\mathrm{CP}$ would then be replied to $a v_{r}$.

Essentially, an appropriate CP-selected is able to efficiently reduce the waiting for a customer when a pick-up is required at the $\mathrm{D} / \mathrm{P}$ spot.

Step 6 Reporting parking reservation: Once notified by GC of the CP-selection decision, $a v_{r}$ informs the GC by sending its reservation, and GC makes that reservation at the selected $\mathrm{CP}$ for the vehicle. Specifically, a parking reservation includes the following information (notations are defined in Table I):

- Identification of the AV

- Expected arrival time (Tav $\left.T_{a v}^{a r r}\right)$

- Expected parking duration ${ }^{3}\left(\theta_{a v}^{p k}\right)$

\footnotetext{
${ }^{3}$ It is assumed as a fixed value in this work, indicating the duration of customer working period
}

TABLE I

LIST OF NOTATIONS

\begin{tabular}{|c|c|}
\hline Symbol & Description \\
\hline$C_{P}$ & Maximum parking capacity of $\mathrm{CP}$ \\
\hline$N_{P}$ & Number of parked AVs at $\mathrm{CP}$ \\
\hline$N_{R}$ & Number of parking reservations at $\mathrm{CP}$ \\
\hline$T_{a v}^{a r r}$ & $\mathrm{AVs}$ arrival time at $\mathrm{CP}$ \\
\hline$\theta_{a v}^{p k}$ & Expected $\mathrm{AV}$ parking period at $\mathrm{CP}$ \\
\hline$T_{\text {cur }}$ & Current time in network \\
\hline$T_{c x}^{t r i p}$ & $\begin{array}{l}\text { AV outbound/inbound trip duration (including customer } \\
\text { walk time) }\end{array}$ \\
\hline$T_{c x}^{v, l_{d p}}$ & $\begin{array}{l}\text { AV travelling time towards the } \mathrm{D} / \mathrm{P} \text { point while customer } \\
\text { is onboard }\end{array}$ \\
\hline$T_{c x}^{l_{d p}, w p}$ & Customer walk time towards WP from the D/P area \\
\hline$T_{c x}^{l_{d p}}$ & $\begin{array}{l}\text { AV travelling time towards next customer destination } \\
\text { from the D/P point }\end{array}$ \\
\hline$T_{a v}^{l_{d p}, c p}$ & $\mathrm{AV}$ travelling time to reach $\mathrm{CP}$ from $\mathrm{D} / \mathrm{P}$ point \\
\hline$T_{a v}^{f i n}$ & Parking finish time point at $\mathrm{CP}$ \\
\hline & Location of a $\mathrm{D} / \mathrm{P}$ point \\
\hline$E W_{a v}^{c p}$ & Expected parking waiting time of $\mathrm{AV}$ at $\mathrm{CP}$ \\
\hline
\end{tabular}

\section{Problem Formulation}

Considering the overall journey experience for AV customers, as well as the efficiency for energy consumption regarding vehicles, we herein aim to achieve the following objectives: a) to minimize the outbound trip (or inbound) ${ }^{4}$ duration for the customer $\left(T_{c x}^{t r i p}\right)$, and $\mathrm{b}$ ) to minimize the remaining travel time for the $\mathrm{AV}$ to continually drive to the park $\left(T_{a v}^{l_{d p}, c p}\right)$. To facilitate the problem formulations, we introduce the following notations:

- $T_{c x}^{v, l_{d p}}$ : the travelling time of $\mathrm{AV}$ towards the $\mathrm{D} / \mathrm{P}$ point $\left(l_{d p}\right)$ while customer is onboard

- $T_{c x}^{l_{d p}, w p}$ : the walk time of the customer towards the destination from the $\mathrm{D} / \mathrm{P}$ area $\left(l_{d p}\right)$

- $\Lambda_{d p}$ : the set of locations of $\mathrm{D} / \mathrm{P}$ in network

- $\Lambda_{c p}$ : the set of CPs in network

Note the first objective is leading to the optimal D/P selection decision-making. Provided the location of $\mathrm{D} / \mathrm{P}$ indexed as $l_{d p}, l_{d p} \in \Lambda_{d p}$, the most appropriate $\mathrm{D} / \mathrm{P}$ spot $\left(l_{d p}^{o p t}\right)$ can be determined by computing the formula as follows.

$$
\begin{aligned}
& \arg \min _{l_{d p} \in \Lambda_{d p}} T_{c x}^{\operatorname{trip}\left(l_{d p}\right)} \\
& :=\left\{l_{d p} \mid l_{d p} \in \Lambda_{d p} \wedge \forall l_{d p}^{i} \in \Lambda_{d p}: T_{c x}^{t r i p\left(l_{d p}^{i}\right)} \geq T_{c x}^{\operatorname{trip}\left(l_{d p}\right)}\right\}
\end{aligned}
$$

s.t.,

$$
\begin{gathered}
T_{c x}^{t r i p\left(l_{d p}\right)}=T_{c x}^{v, l_{d p}}+T_{c x}^{l_{d p}, w p} \\
l_{d p} \in \Lambda_{d p}
\end{gathered}
$$

Note that calculation of $T_{c x}^{\text {trip }\left(l_{d p}\right)}$ according to Eq. (2) derives duration for outbound trip. Regarding the inbound trip duration, calculation is similar, wherein $T_{c x}^{l_{d p}, w p}$ in Eq.

\footnotetext{
${ }^{4}$ Customer outbound trip refers to the journey of $\mathrm{AV}$ travelling to $\mathrm{D} / \mathrm{P}$ point to drop off customer, including customer walk from D/P spot to WP, and inbound trip refers to the trip for $\mathrm{AV}$ picking up customer, including customer walk from WP to D/P spot, plus AV travelling time from that point towards the next customer destination.
} 


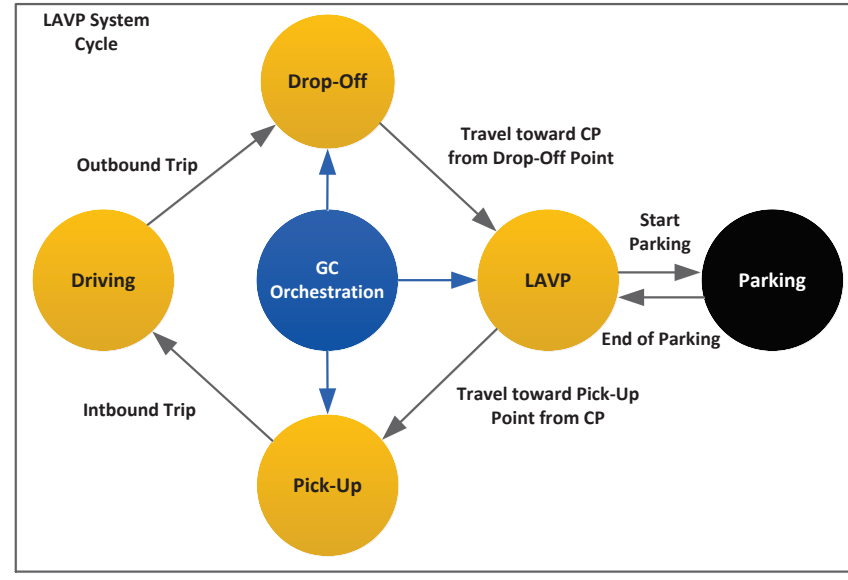

(a)

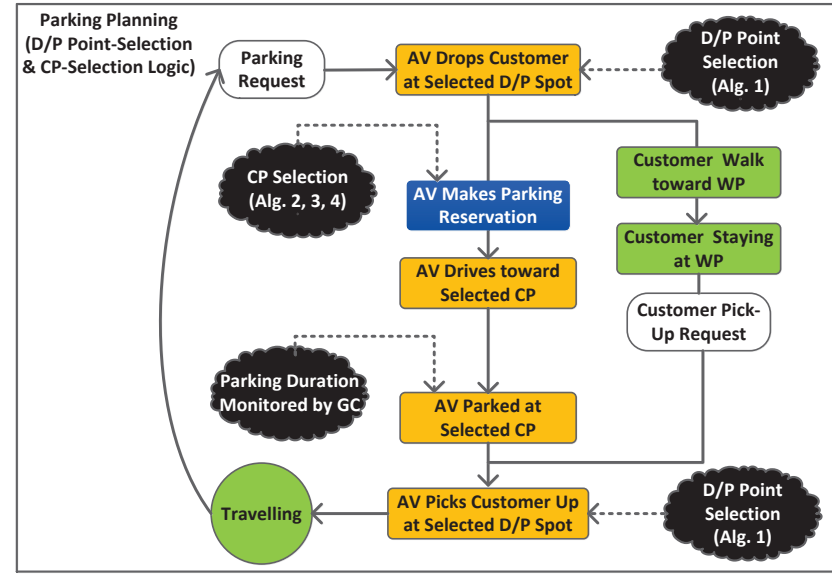

(b)

Fig. 3. System cycle of on-the-move AV LAVP management: (a) System Cycle (b) Operational Logic

(2) is still the walk period for customer, only in reverse direction, and $T_{c x}^{v, l_{d p}}$ in Eq. (2) will be replaced by the value of $T_{c x}^{l_{d p}, d s t}$ that refers to the journey of $\mathrm{AV}$ travelling towards next customer destination from the $\mathrm{D} / \mathrm{P}$ point.

Once the best $\mathrm{D} / \mathrm{P}$ point $\left(l_{d p}^{o p t}\right)$ is confirmed by the $\mathrm{AV}$, the optimal $\mathrm{CP}\left(C P_{o p t}\right)$ can thus be derived by

find $C P_{o p t} \in \Lambda_{c p}$ to minimize $C=\left(T_{a v}^{l_{d p}, c p}+E W_{a v}^{c p}\right)$

where $C$ signifies the cost in relation to $\mathrm{CP}$-selection, and $E W_{a v}^{c p}$ denotes the average time for each AV to wait before parking becomes available. Note Eq. (4) corresponds to the second objective regarding above. According to queueing theory, if the load of parking AVs can be balanced across all $\mathrm{CPs}$, the parking waiting time will be minimized. Such direct correlation between load balancing and service experiences (in terms of waiting time) can be more intuitively demonstrated in latter performance evaluations (Sec. 5.2).

In particular, when $\mathrm{CP}$ not fully occupied, the value of $W_{a v}^{c p}$ equals to 0 . In case that a $\mathrm{CP}$ is fully parked, $W_{a v}^{c p}$ needs to be estimated. The accuracy with respect to the prediction of $W_{a v}^{c p}$ depends on a range of critical context information, including the amount of parked AVs as well as parking reservations. As such, we introduce several algorithms in the following section to facilitate such approximation.

\section{Design OF Scheduling SCHEME FOR RESERVATION ENHANCED LAVP}

Based on previous discussions, the D/P point-selection procedure as well as the $\mathrm{CP}$-selection process will be specifically elaborated by relevant algorithms in this section. Fig. 3 depicts the system cycle of LAVP management with AV on-the-go.

- Driving Phase: The AV is travelling towards its trip destination. This phase is affected by both the customer demand and mobility uncertainty. In particular, it includes the outbound trip when customer is heading to WP, and the inbound trip when customer is heading back home (or elsewhere) from WP.
- Parking Planning Phase: The AV customer requesting car parking while it is within a certain range of the final destination (i.e., WP). AV then queries GC for selection of appropriate $\mathrm{D} / \mathrm{P}$ points and car parks. Based on the locally recorded CPs condition information and D/P spots locations, the GC runs a D/P point-selection logic and a CP-selection logic, respectively. Essentially, the GC globally monitors the CP status and manages AV parking reservations. Such context information is aggregated and updated at GC periodically for effective $\mathrm{CP}$-selection decision making.

- Drop-off Phase: When AV is travelling within a certain range of destination WP, it sends a drop-off request to GC. Upon receiving the request, GC executes the D/P point-selection procedure and returns a proper result. AV accepts the recommendation and drives toward the $\mathrm{D} / \mathrm{P}$ location to drop off the customer, who proceeds to walk to WP, while the vehicle starts to trigger the LAVP phase.

- LAVP Phase: Based on the D/P spot-selected, a proper $\mathrm{CP}$ will be obtained based on the travelling from that $\mathrm{D} / \mathrm{P}$ point, by applying the $\mathrm{CP}$-selection at GC. The AV would then drive in fully autonomous mode towards the $\mathrm{CP}$-selected, and park itself without human interventions at the park.

- Parking Phase: While customer staying at WP, the AV is parked at the CP-selected. Basically, the vehicle would not start to drive unless it is summoned by the customer for a pick-up.

- Pick-up Phase: After work, the customer may send a request to GC for a pick-up. As stated previously in Sec. III, in this paper we assume pick-up spot would be the same as the drop-off place. In case they are different, it can either based on customer demands, or computed by $\mathrm{GC}$ according to the D/P-selection logic. After obtaining the pick-up location from GC, the AV would then start the car and begin the LAVP process to autonomously drive towards the pick-up spot. 


\section{A. D/P Spot-Selection Logic}

By running $T_{c x}^{t r i p\left(l_{d p}\right)}$ for each D/P point in Alg. 1 within an area of $\Lambda_{d p}$ (e.g, within the a radius of $R$ of the final users destination), the one meets the minimum outbound (or inbound) trip duration (according to Eq. (1)) for $a v_{r}$ is selected, and then the GC returns the location of selected D/P point $\left(l_{d p}^{o p t}\right)$ back to $a v_{r}$.

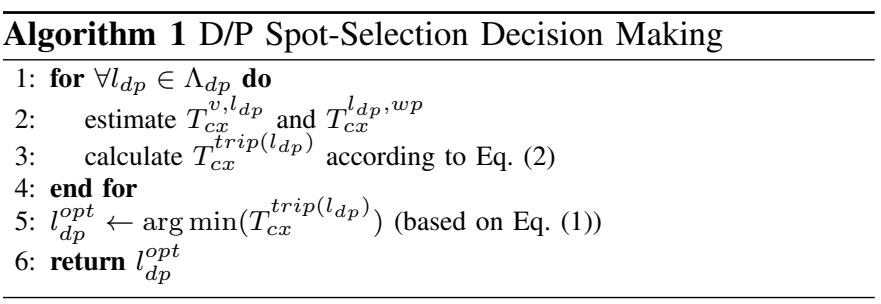

Next, CP-selection decision making process will be discussed. In order to promote such process, an accurate estimation on the value of parking waiting time $\left(E W_{a v}^{c p}\right)$ given by Eq. (4) is required, which leads to the design of a series of algorithms.

\section{B. Available Time for Parking}

Here a list of available times for parking at a CP will be estimated, based on the knowledge of those AVs currently parked as well as parking reservations. Specifically, those parked AVs are characterized as a queue of $N_{P}$, while parking reservations are characterized as a queue of $N_{R}$. In cases that parking space not fully occupied $\left(N_{P}<C_{P}\right)$, the current time (denoted as $T_{\text {cur }}$ ) in the network is estimated as the available time for parking and added to LIST (line 3 in Alg. 2). With those spots already taken, their available parking times are then approximated as $\left(T_{a v_{(j)}}^{a r r}+\theta_{a v_{(j)}}^{p k}\right)$ for each $a v_{(j)}\left(j \in N_{P}\right)$ in line 7 of Alg. 2.

By additionally considering parking reservations $\left(N_{R}\right)$, the LIST can be further refined (from line 9 to line 26 in Alg. 2). The $N_{R}$ is sorted based on First Come First Served (FCFS) discipline, which is in accordance with common practice. Also, LIST is sorted with ascending order and thus, the head value (LIST.GET(0)) refers to the earliest time for parking concerning all parking spots at the CP. Particularly, for those AVs $\left(a v_{k}, k \in N_{R}\right)$ with earlier arrival times than requestor $a v_{r}$, i.e., $\left(T_{a v_{(k)}}^{a r r}<T_{a v_{(r)}}^{a r r}\right)$, the former will lead to the dynamic update of LIST. As such, the earliest available parking spot can be predicted upon the arrival of requestor $a v_{r}$.

- If $T_{a v_{(k)}}^{a r r}$ is earlier than the earliest available parking time, given by ( $\left.L I S T . G E T(0)>T_{a v_{(k)}}^{a r r}\right)$, parking finish time $\left(T_{\left.a v_{(k)}\right)}^{f i n}\right)$ for $a v_{k}$ is calculated as the aggregation of its parking duration $\left(\theta_{a v(k)}^{p k}\right)$ along with the earliest available parking time (line 17 in Alg. 2).

- Otherwise, $a v_{k}$ can be parked right upon its arrival, and the parking finish time $\left(T_{a v_{(k)}}^{f i n}\right)$ is calculated as arrival time plus its parking duration (line 19 in Alg. 2).

When all reserved AVs are considered, provided the condition $\left(T_{a v_{(k)}}^{a r r}<T_{a v_{(r)}}^{a r r}\right)$, the updating on LIST is finished and the list is then returned.

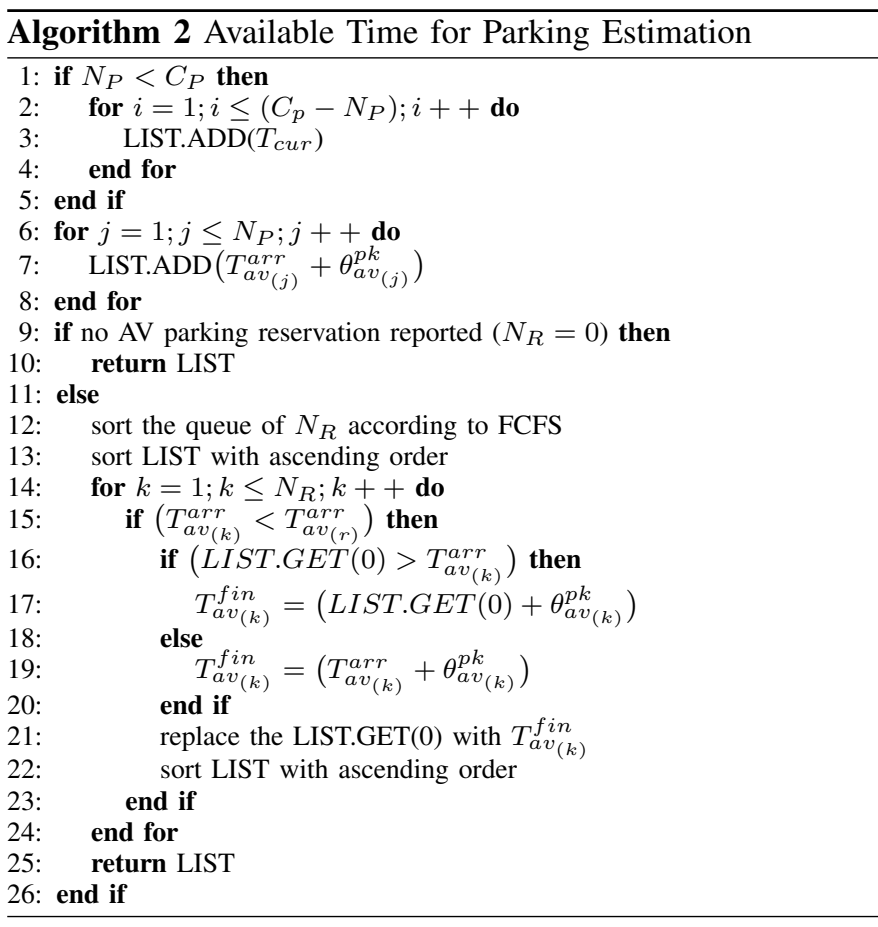

\section{Expected Waiting Time for Parking}

Based on Alg. 2, the expected waiting time for parking $\left(E W_{a v_{(r)}}^{c p}\right)$ of requestor $a v_{r}$ can thus be estimated in Alg. 3.

- If $a v_{r}$ arrives early, provided (LIST.GET(0)> Tav $T_{a(r)}^{a r r}$ ), the value of $E W_{a v_{(r)}}^{c p}$ is calculated as the additional waiting time for the earliest available parking lot (line 3 in Alg. 3).

- Otherwise, the AV can be parked right upon its arrival without waiting (line 5 in Alg. 3)

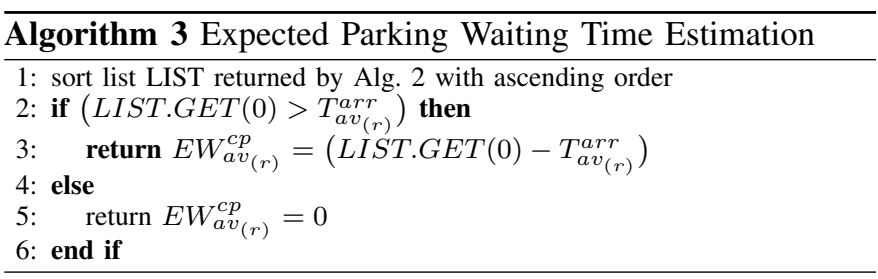

\section{CP-Selection Logic}

By running the cost value $\mathrm{C}$ for each $\mathrm{CP}$ in $\mathrm{Alg}$. 4, the one meets the minimum cost value (according to Eq. (4)) for $a v_{r}$ is selected, and then the GC returns the location of selected $\mathrm{CP}\left(C P_{\text {opt }}\right)$ back to $a v_{r}$.

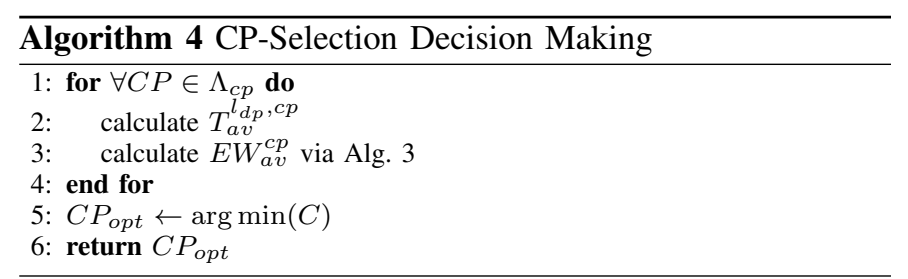




\section{E. Discussion}

1) Privacy Concern: With a centralized management mode, $\mathrm{CP}$-selection as well as D/P point-selection is achieved by a global controller GC as presented in Sec. III-A. For vehicles, $\mathrm{AVs}$ would have to release some status information, such as IDs and locations, so as to enable decision-makings on the optimum selections. As such, the privacy issue could be raised in such case. Usually, one possible solution is to introduce a trustworthy third party by all engaged sides (AVs, CPs and GC) to perform the role of GC, which can be implemented as a cloud server from a reliable platform. Also, encryption techniques (e.g., pseudonym) could be leveraged to hide privacy-sensitive information, such as AV locations and IDs. Although a decentralized approach requires less privacyrelated information via encouraging decision-makings on vehicle side, the communication cost among multiple network entities could become the great concern. In order to assure accuracy pertaining to key information, such as CP status and parking demand load, frequent information exchange are thus required in such distributed manner. The concern with a distributed mode strategy relating to LAVP will be studied in our future work.

2) Reservation Randomness: As presented previously, AV will confirm a $\mathrm{CP}$-selection decision-making by reporting a reservation to the GC, in terms of context including < $a v_{i d}, T_{a v}^{a r r}, \theta_{a v}^{p k}>$. Compared to static context like vehicle IDs and parking durations, expected arrival times of AVs can be quite random. This is mainly due to dynamic traffic situations happening on road in practice, such as traffic jam. Such case would impose the AV mobility uncertainty and thus, arrivals at $\mathrm{CP}$ become unpredictable. Therefore, an update of reservations at GC in a periodical way is important, so as to keep track of any cancellations or modifications on parking reservations. Such consideration in the uncertainty of AV mobility can help to predict future parking states at individual CPs with great accuracy.

3) Parking Pricing Concern: Parking is never really free. Underpricing tends to increase problems such as traffic congestion and pollution, while overpricing leads to revenue loss for car park operators. As such, an appropriate pricing scheme model is important to well balance benefits for all stakeholders. In this work we consider equivalent parking fees for all CPs, since the customer queueing at $\mathrm{CPs}$ and travel cost for $\mathrm{AVs}$ are the major concern for effective CP-selection. While parking cost has been taken into account in some research works [12], how and in what scale it should have an impact on $\mathrm{CP}$-selection decision-making worth further analysis. If price plays an important role, a CP distant from customer D/P point but with lower parking pricing can quickly become a hotspot, while the one in proximity with higher parking pricing but more parking space is less favored. In practice, prices varied from different CPs, and how it can have an impact on the $\mathrm{CP}$-selection needs further research efforts.

4) Electric-Powered $A V$ : Due to advances in sustainable energy development, vehicles have gong electric and start to penetrate the transportation landscape. On the other hand, $\mathrm{AVs}$ are expected to be designed as connected, shared and electrified. Essentially, electric vehicles (EVs) are driving the

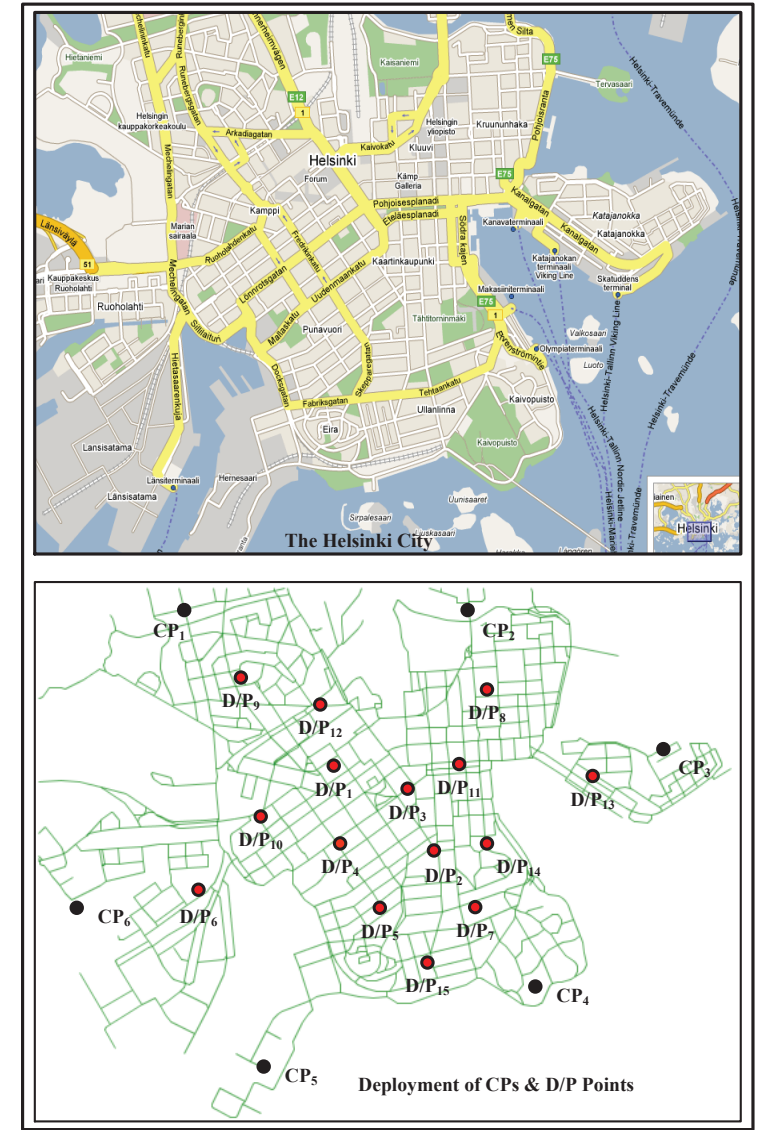

Fig. 4. Simulation scenario of Helsinki City

growth of AVs in many aspects. Environmental impact is one obvious benefit, by integrating renewable energy resources on the power grid. Lower fuel and maintenance fees from electric engine allow for higher usage rates and labor cost, which ultimately drive down transportation cost considerably. Also, electric vehicles are better compatible with $\mathrm{AVs}$ due to their drive-by-wire system built in, replacing traditional mechanical control systems with electronic controls. This creates a more flexible platform for autonomous technologies. Moreover, wireless charging integrates with autonomy seamlessly.

\section{CASE STUdy}

We have implemented a case study under Opportunistic Network Environment (ONE) [20]. In Fig. 4, the default scenario is shown as the downtown area of Helsinki City with $4500 \times 3400 \mathrm{~m}^{2}$ area in Finland. Here, $300 \mathrm{AVs}$ with [30 50] $\mathrm{km} / \mathrm{h}$ variable moving speed are initialized in the network. A total number of $6 \mathrm{CPs}$ and $15 \mathrm{D} / \mathrm{P}$ spots are deployed in the network. Each $\mathrm{CP}$ is with a limited number of 100 parking spots. The final destination (e.g., WP) of every AV customer trip is randomly selected from a location in the map. Particularly, the time for drivers request for drop-off is set as a variable uniformly and randomly selected within the range of [2400s $\sim 600 \mathrm{~s}]$, and 7200s is set as the parking duration (or working period) at $\mathrm{CP}$. The communication cost at the GC side can be calculated as $O\left(N_{a v}\right)$, which is linearly increased by AV density $N_{a v}$. 
Once a parked AV is summoned by a customer, it follows the same route as the outbound trip route back to the $\mathrm{D} / \mathrm{P}$ point, where it picks up the customer and drive towards the next destination. Energy consumption of AV can be calculated according to [21]. Here, the shortest path towards D/P point or $\mathrm{CP}$ is formed for the $\mathrm{AV}$ considering the Helsinki road topology. In reality, the GC is believed to be with super power and super computation capability to make parking/drop-off (or pick-up) plans for all AVs in large scale network. The simulation shows a 12 hours duration experiment with a $0.1 \mathrm{~s}$ resolution.

For comparison, the following schemes are evaluated:

- LAVP-RES: The proposed LAVP service concerning future parking states at individual $\mathrm{CPs}$, wherein the $\mathrm{CP}$ selection decision-making is based on estimations over the parking waiting time (for a future moment) as well as distance between the selected $\mathrm{D} / \mathrm{P}$ area and the $\mathrm{CP}$ (i.e., $\left.C=\left(T_{a v}^{l_{d p}, c p}+E W_{a v}^{c p}\right)\right)$ according to Eq. (4)).

- LAVP-WAI: The proposed LAVP service without concerning future parking states, wherein the decisionmaking on $\mathrm{CP}$-selection is based on local parking waiting time at individual CPs (without considering future reservations).

- LAVP-DST: The LAVP service makes decision on CPselection based on the distance between the selected D/P area and the $\mathrm{CP}$ [11] (i.e., $C=T_{a v}^{l_{d p}, c p}$ ) according to Eq. (4)).

Note that the decision-making on D/P-selection with above schemes are all following the same algorithm of Alg. 1, owing to the optimality according to Euclidean geometry.

Below are the performance attributes that are considered for evaluations:

- Average Parking Waiting Time: The average period between the time an AV arrives at the selected CP and the time it finally gets an available spot for parking. This is the performance metric at system level.

- Average AV Travelling Time: The average time that an AV experiences for its outbound trip, which refers to the journey towards the selected $\mathrm{CP}$, through a $\mathrm{D} / \mathrm{P}$ point. This is the performance at AV side.

- Average Customer Trip Duration: The average time that an AV customer experiences for its outbound trip, from requesting for a drop-off until reaching the final destination, i.e., WP). This is the performance metric at the customer side.

Since in this case study, the inbound trip (from summoning the AV for a pickup to reaching the destination, i.e., home) follows the same route as the outbound. Therefore, it experiences similar duration as the outbound journey. As such, the above performances mainly focus on the outbound trip.

\section{A. Impact of $A V$ Density}

With the rise in AV density, the waiting duration for an available parking spot in a CP can be substantially increased, as shown in Fig. 5 (a). In particular, the proposed LAVP-RES shows its optimality against other schemes, with a considerable reduction on the waiting time. This is mainly benefitted from the capability of enabling predictions on future parking states across the parking network. As shown in the figure, the LAVPWAI endures much longer waiting. Due to limited parking spots, hotspots can easily occur when many AVs constantly choose and drive towards the same CP, especially under LAVPWAI of unpredictable future parking states. It is interesting that such issue becomes much less concerned under the LAVP-DST policy, where $\mathrm{AV}$ s tend to select $\mathrm{CPs}$ geographically close to $\mathrm{D} / \mathrm{P}$ points. This is mainly due to the diversity of customer demand regarding various requirements on $\mathrm{AV}$ destinations that are usually randomly distributed in the network. As such, the CP-selected based on distance (under LAVP-DST) could be widely scattered and thus, congestions at individual CPs are less likely to happen.

Regards the AV travelling duration with varied AV numbers, the performance is shown in Fig. 5 (b). Not surprisingly, the travel period can be greatly reduced by the proposed LAVPRES (and LAVP-DST), by accounting for the travel distance concern. As noticed, the performance seems to be not affected with increased AV density. Similar performance trend also shows in Fig. 5 (c), in terms of customer trip duration. This implies that the AV density has subtle impact on AV/customer travel period. The reason is that these two metrics are mainly governed by the deployment of CPs and D/P points, wherein different numbers of $\mathrm{CPs}$ (or $\mathrm{D} / \mathrm{P}$ spots) could have more influence on the performances (as will be shown in Sec. V.C and Sec. V.D). Particularly in Fig. 5 (c), all schemes experience comparable customer travelling duration. This is owing to the same decision-making on D/P-selection with all schemes following Alg. 1.

\section{B. Impact of Parking Spots}

Here, with increment of the number of parking spots at individual CPs, Fig. 6 (a) shows that the waiting for parking can be greatly reduced under all LAVP schemes. Intuitively, one immediate advantage is to enable estimations on future parking states, which enables great performance gains under LAVP-RES. Great performance can also be achieved under LAVP-DST, similar reasons apply with preceding discussions. As noticed, the waiting is high under LAVP- WAI comparted to other two schemes, although the time is constantly reduced with more parking spots installed. This is mainly due to potential congests happened at CPs, where AVs have to queue up and wait relatively long time for available parking spots.

As discussed previously, it is mainly the deployment of CPs (or D/P points) that finally governs the performance regarding AV travelling duration (and customer travel period). The performance trend is thus not affected with varied parking slots as shown in Fig. 6 (b) and (c), while all schemes performs similarly in Fig. 6 (c), due to same process of $\mathrm{D} / \mathrm{P}$ pointselection adopted by all.

Figure 7 shows the distribution of totally parked AVs among all CPs under different schemes for comparison. Noticeably, the LAVP-RES achieves relatively balanced AV load over all CPs, while LAVP-WAI behaves in a skewed distribution across all CPs. Due to CP-selected potentially scattered over the network, the distribution of parked AVs with LAVP-DST 


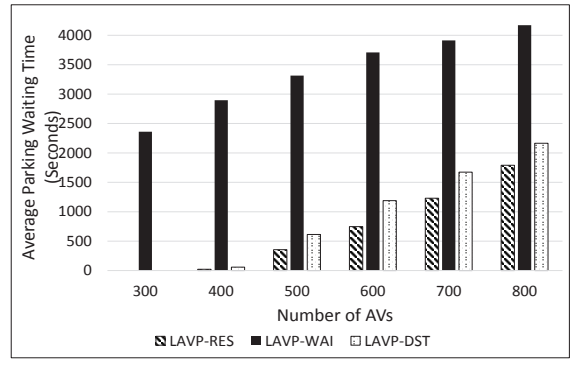

(a)

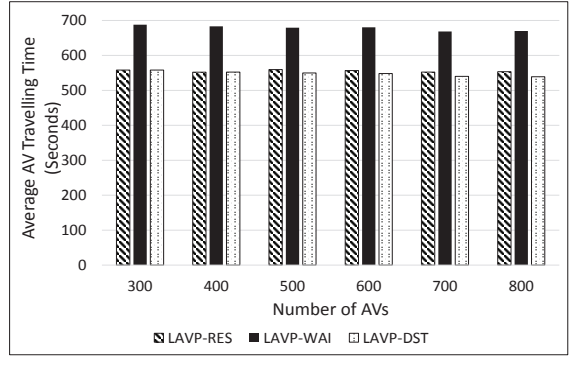

(b)

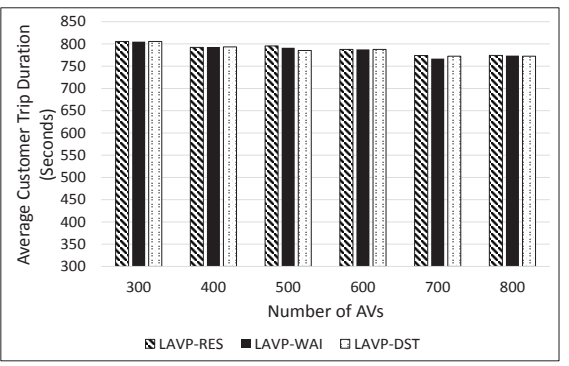

(c)

Fig. 5. Influence of AV Density: (a) Average Parking Waiting Time (b) Average AV Travelling Time (c) Average Customer Trip Duration

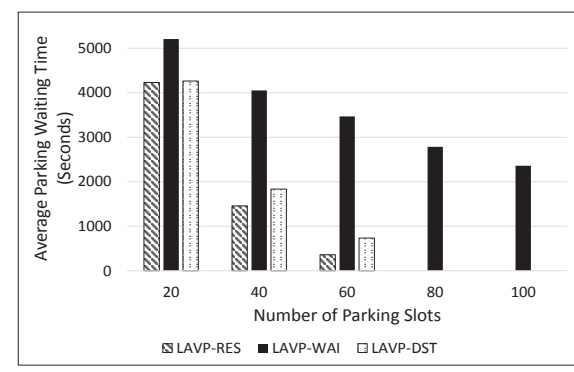

(a)

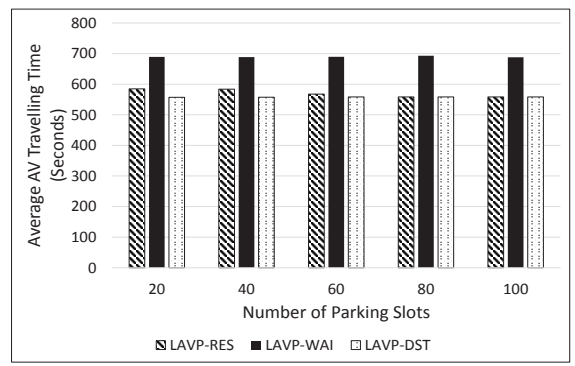

(b)

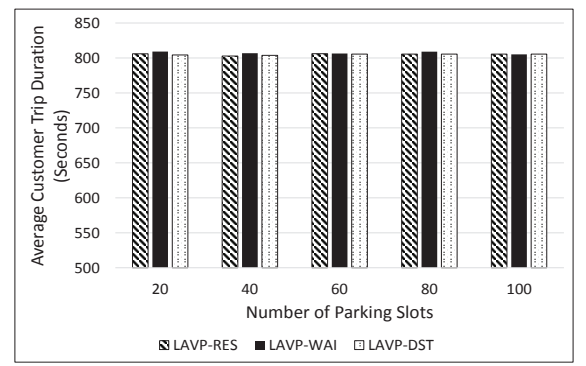

(c)

Fig. 6. Influence of The Number of Parking Spots: (a) Average Parking Waiting Time (b) Average AV Travelling Time (c) Average Customer Trip Duration

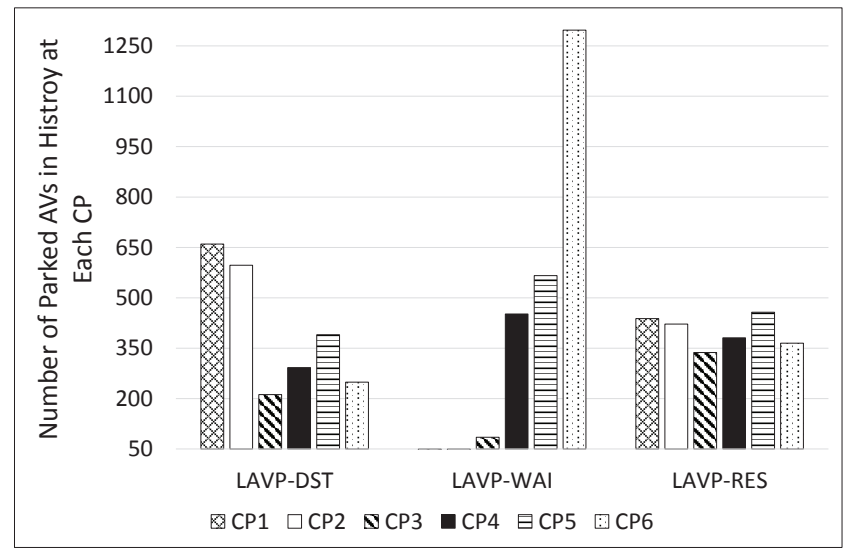

Fig. 7. Distribution of Parked AVs among CPs

displays a moderate skewed distribution against LAVP-WAI. According to the analysis as given in Eq. (4), there is a direct correlation between load balancing and Quality of Experience (QoE), in terms of parking waiting time in this case. Results of Fig. 7 further explains such relationship in an intuitive fashion, along with previous evaluations of Fig. 5 (a) and Fig. 6 (a).

\section{Impact of D/P Points}

Here, we deploy different amount of D/P points with fixed 6 CPs in the network. As shown in Fig. 8, all performances can be improved greatly with more D/P spots installed. Due to a joint concern on parking waiting time as well as travel distance, the LAVP-RES achieves the most performance gains as shown in Fig. 8 (a) and (b), while the LAVP-WAI behaves the worst. When only $4 \mathrm{D} / \mathrm{P}$ points installed, AVs would suffer from relatively longer waiting at CPs as shown in Fig. 8 (a).
The reason is that many AVs tend to select and drive towards the same $\mathrm{CP}$ when they have limited choice on $\mathrm{D} / \mathrm{P}$ points.

Essentially, vehicles needs to stop at appropriate D/P area before heading to CPs, and the amount of AVs could become large travelling through limited D/P points in such case. CPselection could thus be concentrated to a few CPs within distance to the small number of D/P spots. Specifically, all schemes behave similarly with respect to customer travelling period, as shown in Fig. 8 (c), because of the same D/P point-selection process applied to all schemes as discussed previously.

As observed, the improved performances indicate the effectiveness of deployment of D/P points in different scales. Especially in crowded city centers, by deploying more D/P points, parking issues as well as customer QoE (in terms of parking waiting and travelling period, etc.) can be greatly improved.

\section{Impact of CP Number}

With 15 D/P points fixed, we show in Fig. 8 the influence of CP deployment in performances. As noticed in Fig. 9 (a), all schemes suffer from high parking waiting time with only $1 \mathrm{CP}$ deployed. This is mainly due to limited choice on CPs over the network. Such undesired effects would be greatly relieved when more CPs installed, i.e., from 3 increased to 6 CPs. Obviously, the LAVP-RES achieves the best performance against all other schemes, benefited from estimation capability in future parking states. Not surprisingly, the LAVP-WAI experiences the worst performance, while LAVP-DST behaves desirably, which is in accordance with results in Fig. 5, 6 and 8 (a) and (b). 


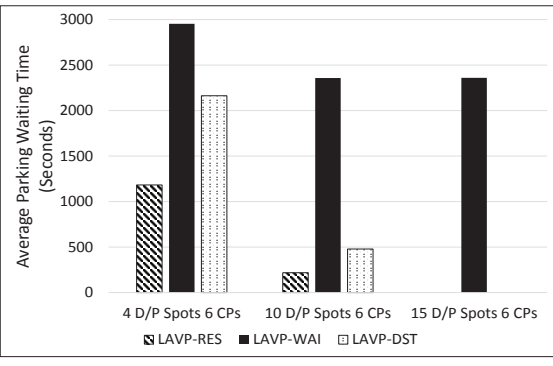

(a)

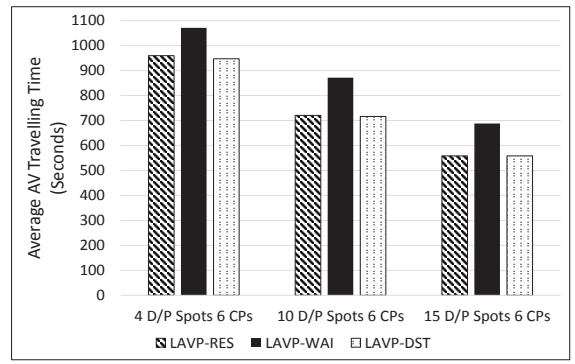

(b)

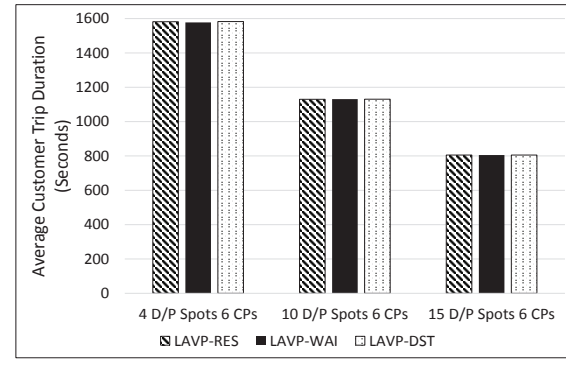

(c)

Fig. 8. Influence of Deployment of D/P Points: (a) Average Parking Waiting Time (b) Average AV Travelling Time (c) Average Customer Trip Duration

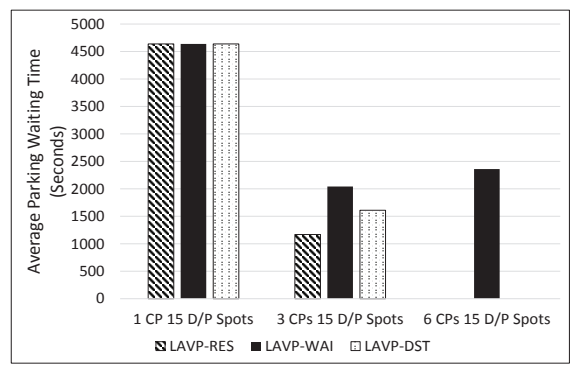

(a)

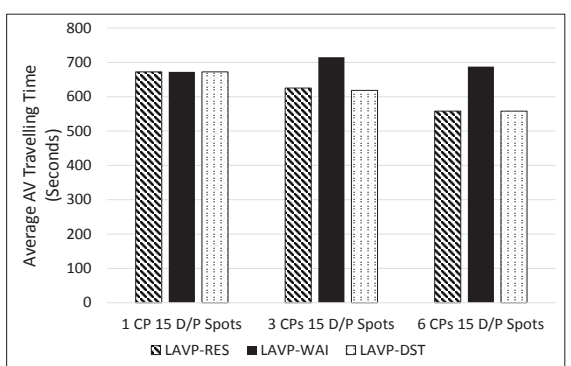

(b)

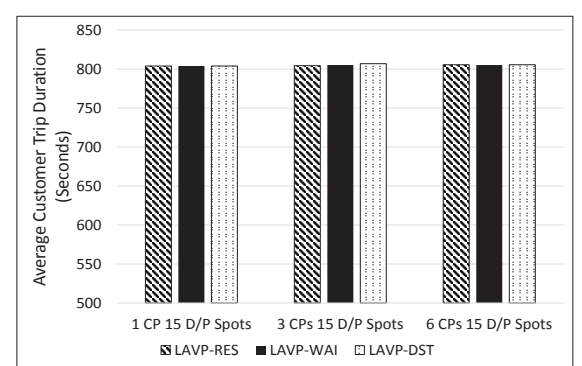

(c)

Fig. 9. Influence of Deployment of CPs: (a) Average Parking Waiting Time (b) Average AV Travelling Time (c) Average Customer Trip Duration

As for the AV travelling period, all schemes experiences reduced AV travel with more CPs deployed as shown in Fig. 9 (b), except for the LAVP-WAI strategy, which seems to be not affected. This implies that CPs would be highly concentrated with AVs under LAVP-WAI scheme, wherein $\mathrm{CP}$-selection is solely based on local parking states, oblivious of future parking situations. As such, the CP-selected could become overcrowded when loads of AVs have the same choice. Particularly, the customer travel is not influenced as observed in Fig. 9 (c), and keeps stable with varied CP number. This indicates that the $\mathrm{CP}$ deployment does not have a critical play in the customer travelling duration, given the number of $\mathrm{D} / \mathrm{P}$ spots are fixedly installed.

\section{CONCLUSION}

In this paper, we focus on major concerns within the LAVP context, with respect to where to park as well as where to drop off (or pick-up) upon customer demands during their journeys. In order to facilitate the intelligent CP-selection (and D/P point-selection) process, a reservation-based LAVP service framework is then proposed. Considering the practicality concern in limited parking space at CPs, the LAVP service is further enhanced with parking reservations. By enabling accurate estimations on parking states for a future moment, the parking load can be accurately anticipated at a particular $\mathrm{CP}$, thereby avoiding hotspots for $\mathrm{AV}$ parking. Comprehensive simulation studies are executed to evaluate the viability of the proposed framework. By comparing to other benchmarks, results show the viability of the proposed scheme for determining an optimal CP-selection (and D/P spot-selection). Meanwhile, the parking load can be well balanced over all CPs based on the proposed framework.

\section{REFERENCES}

[1] J. Contreras-Castillo, S. Zeadally, and J. A. G. Ibanez, Solving vehicular ad hoc network challenges with big data solutions, 3rd ed. IET Netw., vol. 5, no. 4, pp. 81C84, Jul. 2016.

[2] M. Gross, A planet with two billion cars, 3rd ed. Current Biol., vol. 26, no. 8, pp. R307CR310, 2016.

[3] Hussain, R. and Zeadally, S., Autonomous Cars: Research Results, Issues, and Future Challenges, 3rd ed. IEEE Communications Surveys \& Tutorials, vol. 21, no. 2, pp.1275-1313, 2018.

[4] Chirca, M., Chapuis, R. and Lenain, R., Autonomous valet parking system architecture, 3rd ed. in IEEE International Conference on Intelligent Transportation Systems, 2015.

[5] Mingardo, G., van Wee, B., and Rye, T., Urban parking policy in Europe: A conceptualization of past and possible future trends, 3rd ed. Transportation Research Part A: Policy and Practice, vol. 74, pp. 268-281, 2015.

[6] Du, X., and Tan, K. K., Autonomous reverse parking system based on robust path generation and improved sliding mode control, $3 \mathrm{rd}$ ed. IEEE Transactions on Intelligent Transportation Systems, vol. 16, no. 3, pp. 1225-1237, 2014.

[7] Schwesinger, Ulrich, et al., Automated valet parking and charging for e-mobility, 3rd ed. IEEE Intelligent Vehicles Symposium (IV), 2016.

[8] Banzhaf, Holger, et al., The future of parking: A survey on automated valet parking with an outlook on high density parking, 3rd ed. IEEE Intelligent Vehicles Symposium (IV), 2017.

[9] S. Zeadally, R. Hunt, Y.-S. Chen, A. Irwin, and A. Hassan, Vehicular ad hoc networks (VANETS): Status, results, and challenges, 3rd ed. Telecommun. Syst., vol. 50, no. 4, pp. 217C241, Aug. 2012.

[10] Min, Kyoungwook, et al., Design and implementation of path generation algorithm for controlling autonomous driving and parking, 3rd ed. 12th IEEE International Conference on Control, Automation and Systems, 2012.

[11] Khalid, M., Cao, Y., Zhang, X., Han, C., Peng, L., Aslam, N., and Ahmad, N., Towards autonomy: Cost-effective scheduling for long-range autonomous valet parking (lavp), 3rd ed. in IEEE Wireless Communications and Networking Conference (WCNC), 2018.

[12] Khalid, M., et al., AVPark: Reservation and Cost Optimization Based Cyber-Physical System for Long-Range Autonomous Valet Parking ( $L$ AVP), 3rd ed. IEEE Access, vol. 7, pp. 114141-114153, 2019.

[13] J. A. Guerrero-Ibanez, S. Zeadally, and J. Contreras-Castillo, Integration challenges of intelligent transportation systems with connected vehicle, 
cloud computing, and Internet of Things technologies, 3rd ed. IEEE Wireless Commun., vol. 22, no. 6, pp. 122C128, Dec. 2015.

[14] J. Contreras-Castillo, S. Zeadally, and J. A. G. Ibá ñez, A seven-layered model architecture for Internet of Vehicles, 3rd ed. J. Inf. Telecommun., vol. 1, no. 1, pp. 4C22, 2017.

[15] Song, B., Cooperative lateral vehicle control for autonomous valet parking, 3rd ed. International Journal of Automotive Technology, vol. 14, no. 4, pp. 633-640, 2013.

[16] [online 2020] Waymo, www.waymo.com

[17] [online 2019] Global Self-Driving Car Market Forecast to 2027 Featuring Featuring Audi, BMW, Ford, Toyota, General Motor, Tesla, https://www.prnewswire.com/news-releases/global-self-driving-carmarket-forecast-to-2027-featuring-audi-bmw-ford-toyota-general-motortesla-300956607.html

[18] [online 2019] Autonomous cars to grow to 24 million units in AsiaPacific by 2024, https://auto.economictimes.indiatimes.com/news/autotechnology/autonomous-cars-to-growth-to-24-million-units-in-asiapacific-by-2024/70701274

[19] [online 2019] Driverless in the Parking Lot. Autonomous Valet Parking, https://www.daimler.com/innovation/case/autonomous/driverlessparking.html

[20] A. Keränen et al., The ONE Simulator for DTN Protocol Evaluation, 3rd ed. in ICST SIMUTools 09, Rome, Italy, March, 2009.

[21] M. Zhou, H. Jin, and W. Wang, A review of vehicle fuel consumption models to evaluate eco-driving and eco-routing, 3rd ed. Transportation Research Part D: Transport and Environment, vol. 49, no. 12, pp. 203218, Dec. 2016.

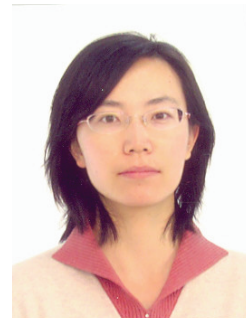

smart grid, etc.

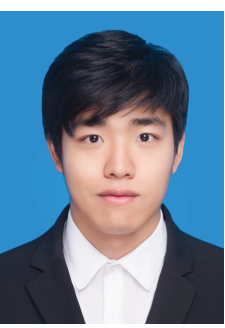

Shuohan Liu received the MSc degree from the School of Computing, at Newcastle University, UK in 2018. He is currently the PhD student at School of Computing and Communication, Lancaster University, UK. His research interests focus on Intelligent Transport Systems.

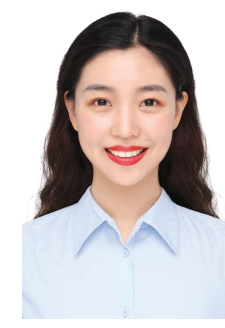

Xu Zhang received her B.Eng. degrees in telecommunication engineering from Xidian University, $\mathrm{X}$ i'an, China, in 2007 and M.Sc. from University of Science and Technology, Beijing, China, in 2010, respectively. She obtained her Ph.D. degree from University of Surrey, Surrey, U.K. in 2015. She is currently a Lecturer with the Department of Computer Science, Xi' an University of Technology, Xi'an, China. Her research interests include Networking and Network Management, including aspects such as content deliver techniques, edge computing, and

Yuan Fang received her bachelor degree from the institution for transportation engineering at Dalian maritime university, China in 2014. She is currently studying for master degree at school of vehicle engineering, Dalian university of technology, China. Her research interests focus on intelligent transport systems.

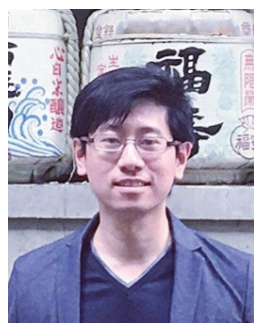

Yue Cao received his $\mathrm{PhD}$ degree from the Institute for Communication Systems (ICS) formerly known as Centre for Communication Systems Research, at University of Surrey, Guildford, UK in 2013. Further to his PhD study, he had conducted research fellow at University of Surrey, and academic faculty at Northumbria University, Lancaster University, UK and Beihang University, China; and he is currently the Professor at School of Cyber Science and Engineering, Wuhan University, China. His research interests focus on Intelligent Transport Systems, including E-Mobility, V2X, Edge Computing. 